\title{
Spongia (Heterofibria) catarinensis sp. nov. (Porifera, Spongiidae) no litoral de Santa Catarina, Brasil
}

\author{
Beatriz Mothes ${ }^{1,4}$, Gustavo Leite Kasper ${ }^{1,6}$, Cléa Lerner ${ }^{1,4}$, Maurício Campos ${ }^{2,5}$ \& João Luís Carraro ${ }^{3,4}$ \\ 1. Museu de Ciências Naturais, Fundação Zoobotânica do Rio Grande do Sul, Rua Dr. Salvador França, 1427, 90690-000 Porto Alegre, RS, \\ Brasil. \\ 2. Programa de Pós-Graduação em Biologia Animal, UFRGS \\ 3. Programa de Pós-Graduação em Ecologia, UFRGS. \\ 4. Bolsista CNPq. \\ 5. Bolsista CAPES. \\ 6. Bolsista PIBIC-CNPq.
}

\begin{abstract}
Spongia (Heterofibria) catarinensis sp. nov. (Porifera, Spongiidae) at the coastline of Santa Catarina, Brazil. A new species, Spongia (Heterofibria) catarinensis, is described from Ilha das Aranhas $\left(27^{\circ} 29^{\prime} 077^{\prime \prime}\right.$ 'S, 48 $21^{\circ} 380^{\prime \prime}$ ), State of Santa Catarina, Brazil. It differs from the other southwestern Atlantic species of this genus by external morphology and architecture. The new species is characterized for presenting massive form, incrusting with lobate projections; primary fibres measuring $60-100 \mu \mathrm{m}$; secondary fibres 11,5-69 $\mu \mathrm{m}$; pseudotertiary fibres 2,3-23 $\mu \mathrm{m}$ and distance between primary fibres 391-920 $\mu \mathrm{m}$; distance between secondary/pseudotertiary fibres $92-575 \mu \mathrm{m}$; secondary/pseudotertiary fibres constituting polygonal meshes 30-700 $\mu \mathrm{m}$ diameter. This species belongs to subgenus Heterofibria Cook \& Bergquist, 2001 for displaying a clear dichotomy of its fibres.
\end{abstract}

KEYWORDS. Spongia, new species, taxonomy, southwestern Atlantic, Brazilian coast.

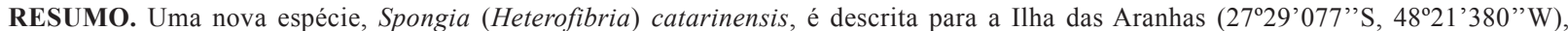
Estado de Santa Catarina, Brasil. Difere das outras espécies do gênero do Atlântico sudoeste pela morfologia externa e arquitetura. A nova espécie é caracterizada por apresentar forma massiva, incrustante com projeções lobulares; fibras primárias medindo $60-100 \mu \mathrm{m}$; fibras secundárias 11,5-69 $\mu \mathrm{m}$; fibras pseudoterciárias 2,3-23 $\mu \mathrm{m}$ e distância entre fibras primárias 391-920 $\mu \mathrm{m}$; distância entre fibras secundárias/pseudoterciárias 92-575 $\mu \mathrm{m}$; fibras secundárias/pseudoterciárias constituindo malhas poligonais com 30-700 $\mu \mathrm{m}$ de diâmetro. A espécie pertence ao subgênero Heterofibria Cook \& Bergquist, 2001 por apresentar uma clara dicotomia de suas fibras.

PALAVRAS-CHAVE. Spongia, nova espécie, taxonomia, Atlântico Sudoeste, costa brasileira.

Raros são os estudos taxonômicos com espécies do gênero Spongia Linnaeus, 1759 na costa brasileira. É importante ressaltar que o gênero Euspongia Bronn, 1859 foi sinonimizado com Spongia (COOK \& BERGQUIST, 2001). O elenco de espécies conhecidas, válidas até o momento, foi estudado e registrado somente no século XIX: HYATT (1877) citou para Fernando de Noronha Spongia officinalis tubulifera var. pertusa Hyatt, 1877; Spongia equina meandriniformis Duchassaing \& Michelotti, 1864 e Spongia vermiculata cookii var. ditelliformis Hyatt, 1877; POLÉJAEFF (1884) descreveu Euspongia officinalis var. lobosa para águas rasas da Bahia; LENDENFELD (1889) citou para a "Província de Pernambuco" Euspongia trincomalensis (Hyatt, 1877), incluindo na sinonímia Spongia officinalis tubulifera var. mollis Hyatt, 1877; nesta ocasião também confirmou o registro de Euspongia officinalis var. lobosa Poléjaeff, 1884 para a costa baiana. Boury-Esnault (1973) descreveu Spongia bresiliana para Pernambuco, Alagoas, Bahia e Rio de Janeiro e citou Spongia virgultosa (Schmidt, 1868) para Pernambuco e Bahia.

VAn Soest (1978), ao estudar espécies do Caribe, propôs uma nova combinação, Spongia pertusa (Hyatt, 1877), sinonimizando a esta espécie Spongia officinalis tubulifera var. pertusa e $S$. officinalis tubulifera var. mollis, ambas de Hyatt, 1877; também nesta obra, sinonimizou S. vermiculata Duchassaing \& Michelotti,
1864 a S. barbara Duchassaing \& Michelotti, 1864. $\mathrm{Na}$ literatura são citadas para o Brasil Spongia barbara (HyATt, 1877 como $S$. vermiculata); S. officinalis (Hyatt, 1877; PoléJaefF, 1884 como var. lobosa) (Lendenfeld, 1889 como S. officinalis lobosa); . trincomalensis Lendenfeld, 1889 e $S$. vermiculata cookii ditelliformis. VAN SoEst (1978) registra que tanto $S$. bresiliana como S. virgultosa sensu Boury-Esnault, 1973 são na verdade Hyattella intestinalis (Lamarck, 1814). Mais recentemente, o gênero Euspongia Bronn, 1859 foi sinonimizado com Spongia por CoOK \& BERGQUIST (2001)

Uma revisão das espécies do gênero Spongia na costa brasileira é necessária, objetivando o conhecimento do número de espécies válidas, bem como sua inclusão nos respectivos subgêneros.

Objetiva-se a descrição de uma nova espécie, oferecendo pela primeira vez fotos in situ de um representante do gênero Spongia na costa brasileira.

\section{MATERIAL E MÉTODOS}

O espécime foi detectado na costa do Estado de Santa Catarina, Ilha das Aranhas $\left(27^{\circ} 29^{\prime} 077^{\prime}\right.$ 'S, $\left.48^{\circ} 21^{\prime} 380^{\prime \prime} \mathrm{W}\right)$, a $13 \mathrm{~m}$ de profundidade. A coleta foi realizada através de mergulho autônomo, retirando-se uma pequena amostra da esponja com o auxílio de faca. O espécime foi fotografado in situ com câmera fotográfica 
equipada com lente $60 \mathrm{~mm}$, caixa estanque e dois flashes. A amostra coletada foi conservada em álcool $96^{\circ} \mathrm{GL}$ e está depositada na Coleção de Poríferos Marinhos do Museu de Ciências Naturais da Fundação Zoobotânica do Rio Grande do Sul.

A metodologia utilizada para preparação de cortes espessos objetivando a observação da estrutura do esqueleto segue MотнEs et al. (2004). Para o estudo de fibras isoladas, destacaram-se de um fragmento sob microscópio estereoscópico e, com auxílio de pinça e agulha histológica, retiraram-se algumas fibras de espongina, as quais foram depositadas sobre lâmina e cobertas com resina composta de polímeros em xileno e lamínula.

Microfotografias das fibras foram realizadas com microscópio óptico equipado com câmera digital. Mensurações das fibras referem-se à mínima, média, máxima, expressas em $\mu \mathrm{m}, \mathrm{N}=50$.

As abreviaturas utilizadas correspondem a: $\mathrm{MCN}$, Museu de Ciências Naturais, Fundação Zoobotânica do Rio Grande do Sul, Porto Alegre; MCNPOR, coleção de Porifera do MCN; MO, Microscópio óptico; SDCC/NZ, coleção particular Steve de C. Cook (destinado para National Museum of New Zealand); ZMA, Zoölogisch Museum Amsterdam, Amsterdam; ZMB, Zoologisches Museum für Naturkunde an der Universität Humboldt zu Berlin, Berlin.

\section{Spongia (Heterofibria) catarinensis Mothes \& Lerner sp. nov. (Figs. 1-6)}

Etimologia. O nome específico refere-se à Santa Catarina, estado brasileiro onde está a localidade-tipo, Ilha das Aranhas.

Material-tipo. Holótipo, BRASIL, Santa Catarina: Ilha

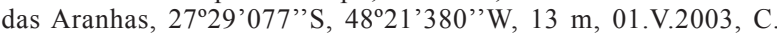
Lerner \& J. P. Cauduro Filho leg. (MCNPOR 5998).

Material adicional examinado (lâminas). Spongia tubulifera Lamarck, 1814 (ZMAPOR 17576); Spongia magellanica Thiele, 1905 (holótipo ZMB 3338); Spongia (Australospongia) gracilis Cook \& Bergquist, 2001 (holótipo SDCC/NZ 115, esquizoholótipo MCNPOR 6899); Spongia (Heterofibria) cristata Cook \& Bergquist, 2001 (SDCC/NZ 043, MCNPOR 6900).

Descrição (Figs. 1-4). Esponja maciça incrustante com projeções irregulares espalhadas lateralmente. Superfície com discretas projeções lobadas elevadas. Ósculos em geral situados na porção mais alta destas projeções, muitos deles dispostos discretamente de modo linear (maior ósculo observado 0,2 cm), circulares, com uma discreta membrana translúcida que circunda a abertura. Poros não observados. Superfície com discretos microcônulos (altura 0,2 cm) de onde protraem fibras primárias, responsáveis pela aspereza da esponja ao tato. Derme destacável in situ e rígida e não-destacável ex situ. Consistência compressível e elástica in situ e ex situ. Coloração in situ roxo-escura externamente e bege internamente; ex situ marrom-escura e amarelo-escura. Dimensões do fragmento ex situ: 7,0 x 5,5 x 0,9 cm.
Esqueleto (Figs. 5-6). Arquitetura do esqueleto fibrosa, pouco densa. Fibras primárias ascendentes preenchidas esparsamente com material exógeno, mais evidentes próximas à superfície, dispostas perpendicularmente, algumas discretamente radiais em relação à superfície. Fibras secundárias e pseudoterciárias sem preenchimento, dominantes no esqueleto. Fibras secundárias e pseudoterciárias são interconectantes com as primárias. Coloração das fibras: amarelo-clara, quase transparente (em luz transmitida) e branco-amarelada a olho nu. Diâmetro das fibras: primárias 60-69,3-100; secundárias 11,5-32,2-69; pseudoterciárias 2,3-13,3-23. Distância entre fibras: primárias 391-676,2-920; secundárias/pseudoterciárias: 92-304,1-575. Retículo pouco denso. Fibras secundárias/pseudoterciárias constituem malhas poligonais de 30-700 $\mu \mathrm{m}$ de diâmetro.

Discussão. O espécime foi coletado na Província Paulista (PALÁCIO, 1982), uma notável área de transição entre a Província Brasileira e a Província Patagônica, caracterizada por espécies endêmicas, comuns à área que se estende desde o Rio de Janeiro até Santa Catarina (LERNER \& HAJdU, 2002; LeRNer et al., 2005). Apesar desse evidente endemismo, alguns poríferos tropicais têm seu limite meridional no infralitoral da costa catarinense (Mothes-De-Moraes, 1987; Mothes \& Lerner, 1994; LERNER, 1996; LERNER et al., 2005). Por sua vez, esta região ao sul também é limítrofe com águas frias vindas do sul da América do Sul (Corrente das Malvinas) e com forte aporte de estoque faunístico bentônico de origem antártico/magelânico.

Ao examinar as fotos do espécime in situ e ex situ, bem como a arquitetura do esqueleto, Rob Van Soest considerou que não seria impossível ser coespecífica com S. tubulifera, citada para Curaçao e Bonaire, Caribe (VAN SoEST, 1978). Entretanto, afirma que as elevações lobulares são normalmente mais altas e acentuadamente mais tubulares em S. tubulifera, além da coloração preta. Considerou também a possibilidade de ser co-específica com Spongia magellanica Thiele, 1905, espécie de águas frias e rasas, descrita para o Pacífico sul (Calbuco, Chile) e conhecida também no Atlântico Sul ocidental para Mar del Plata, Argentina (BurTon, 1940).

O exame comparativo da arquitetura do esqueleto de $S$. tubulifera revelou ser esta distinta da nova espécie por apresentar fibras secundárias mais estreitas, malhas menores e ausência de fibras pseudo-secundárias/ terciárias (Fig. 7). Por sua vez, S. magellanica apresenta fibras primárias de diâmetro maior, com maior distância umas das outras e contendo elevada quantidade de material exógeno; também não foi observada a ocorrência de um grupo de fibras menores do que as secundárias (Fig. 8). A identificação do subgênero foi feita com base no exame do material de Spongia (Heterofibria) cristata Cook \& Bergquist, 2001.

Após ter examinado a foto da arquitetura do esqueleto da nova espécie, Steve de C. Cook confirmou tratar-se deste subgênero pela aparente dicotomia das fibras subprimárias, que se dividem em secundárias e pesudoterciárias (COOK \& BERGQUIST, 2001). As espécies do subgênero Heterofibria Cook \& Bergquist, 2001 são 

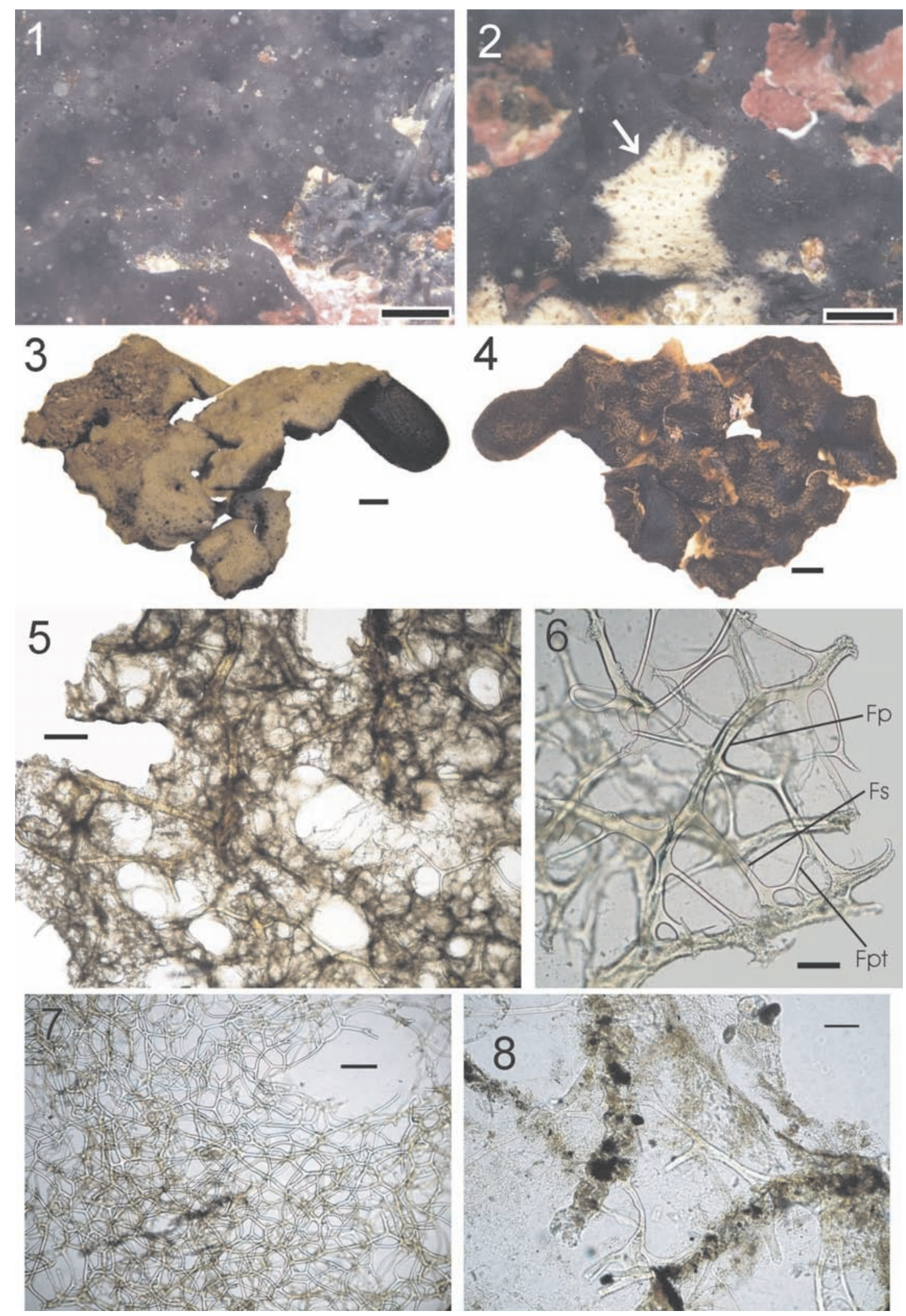

Figs. 1-8. Spongia (Heterofibria) catarinensis sp. nov: 1, espécime in situ (escala, $2 \mathrm{~cm}$ ); 2, espécime in situ, seta indicando porção interna (escala, $1 \mathrm{~cm}$ ); 3, espécime ex situ, vista interna (escala, $1 \mathrm{~cm}$ ); 4, espécime ex situ, vista superior (escala, $2 \mathrm{~cm}) ; 5$, MO, arquitetura do esqueleto em corte perpendicular (escala, $200 \mu \mathrm{m}) ; 6, \mathrm{MO}$, arquitetura do esqueleto de fibras isoladas (Fp, fibra primária; Fpt, fibra pseudoterciária/terciária; Fs, fibra secundária) (escala, $100 \mu \mathrm{m}$ ); 7, Spongia tubulifera Lamarck, 1814: MO, arquitetura do esqueleto de fibras isoladas (escala, $200 \mu \mathrm{m}$ ); 8, Spongia magellanica Thiele, 1905: MO, arquitetura do esqueleto de fibras isoladas (escala, $200 \mu \mathrm{m})$. 
conhecidas apenas para a costa da Nova Zelândia (Cook \& BERGQUIST, 2001) e, por estarem em áreas geográficas disjuntas e em província biogeográfica distinta, não foram comparadas com a nova espécie.

Agradecimentos. Ao Dr. Rob Van Soest (ZMA) e ao Dr. Steve de C. Cook (Department of Anatomy, School of Medicine, University of Auckland, New Zealand), pelas valiosas contribuições na identificação da espécie; a João Paulo Cauduro Filho pelo apoio durante as coletas e pela foto do exemplar in situ; ao $\mathrm{CNPq}$ e CAPES, pela concessão das bolsas. Este trabalho recebeu suporte financeiro do $\mathrm{CNPq}$ Processo n. ${ }^{\circ}$ 52.0858/99-9.

\section{REFERÊNCIAS BIBLIOGRÁFICAS}

Boury-Esnault, N. 1973. Campagne de la 'Calypso' au large des côtes atlantiques de l'Amérique du Sud (1961-1962). I. 29. Spongiaires. Résultats Scientifiques des Campagnes de la "Calypso" 10:263-295.

Burton, M. 1940. Las Esponjas marinas del Museo Argentino de Ciencias Naturales (Parte 1). Anales del Museo Argentino de Ciencias Naturales "Bernardino Rivadavia" 40(6): 95-121.

Cook, S. de C. \& Bergquist, P. 2001. New species of Spongia (Porifera: Demospongiae: Dictyoceratida) from New Zealand, and a proposed subgenus structure. New Zealand Journal of Marine and Freshwater Research 35:33-58.

Hyatт, A. 1877. Revision of the North American Poriferae; with remarks upon foreign species. Part II. Memoirs of the Boston Society of Natural History 2(5):481-554.

LENDENFELD, R. Von. 1889. A monograph of the horny sponges.
London, Trübner. 936p.

Lerner, C. B. 1996. Esponjas da Ilha da Galé (Porifera, Demospongiae), Reserva Biológica Marinha do Arvoredo, Santa Catarina, Brasil. Biociências 4(2):101-124.

Lerner, C. B. \& Hajdu, E. 2002. Two new Mycale (Naviculina) (Mycalidae, Poecilosclerida, Demospongiae) from the Paulista Biogeographic Province (SW Atlantic). Revista Brasileira de Zoologia 19(1):109-122.

Lerner, C. B.; Mothes, B. \& Carraro, J. L. 2005. Novos registros e ampliação de limites meridionais de distribuição de poríferos (Porifera, Demospongiae) no Atlântico sudoeste. Revista Brasileira de Zoologia 22(3):596-612.

Mothes, B. \& Lerner, C. B. 1994. Esponjas marinhas do infralitoral de Bombinhas (Santa Catarina, Brasil) com descrição de três espécies novas (Porifera: Calcarea e Demospongiae). Biociências 2(1):47-62.

Mothes, B.; Campos, M.; Lerner, C. B. \& Ferreira-Correia, M. M. 2004. Esponjas (Demospongiae, Halichondrida) da costa do Maranhão, Brasil. Iheringia, Série Zoologia, 94(2):149-154. Mothes-de-Moraes, B. 1987. Ocorrência de poríferos na zona de maré da Ilha de João da Cunha, Porto Belo, Santa Catarina, Brasil (Porifera - Demospongiae). Iheringia, Série Zoologia, (66):129-139.PALÁCIO, F. J. 1982. Revisión zoogeográfica marina del sur del Brasil. Boletim do Instituto Oceanográfico da Universidade de São Paulo 31(1):69-92.

PoléJaefF, N. 1884. Report on the keratosa collected by H. M. S. Challenger during the years 1873-76. Report on the scientific results of the voyage of $H$. M. S. Challenger during the years 1873-76. Zoology 11:1-88.

Van Soest, R. W. M. 1978. Marine sponges from Curaçao and other Caribbean localities. Part I. Keratosa. Studies on the fauna of Curaça and other Caribbean Islands 56(179):1-94. 